\title{
Seventy new non-eclipsing BEER binaries discovered in CoRoT lightcurves and confirmed by RVs from AAOmega
}

\author{
Lev Tal-Or ${ }^{\text {a }}$, Simchon Faigler, and Tsevi Mazeh \\ School of Physics and Astronomy, Raymond and Beverly Sackler Faculty of Exact Sciences, Tel Aviv \\ University, Israel

\begin{abstract}
We applied the BEER algorithm to the CoRoT lightcurves from the first five LRc fields and identified 481 non-eclipsing BEER candidates with periodic lightcurve modulations and amplitudes of $0.5-87 \mathrm{mmag}$. Medium-resolution spectra of $281 \mathrm{can}-$ didates were obtained in a seven-night AAOmega radial-velocity (RV) campaign, with a precision of $\sim 1 \mathrm{~km} / \mathrm{s}$. The RVs confirmed the binarity of 70 of the BEER candidates, with periods of $0.3-10$ days.
\end{abstract}

\section{Introduction}

BEER [1] is a new method to discover short-period non-eclipsing binaries by using precise photometric lightcurves obtained with space missions like CoRoT and Kepler. The BEER algorithm searches for stars that show in their lightcurves a combination of the BEaming, Ellipsoidal, and Reflection modulations induced by a short-period companion.

Recently we have reported several discoveries made by applying the BEER algorithm to Kepler data - seven new binaries with low-mass companions [2], and a hot Jupiter with evidence of superrotation $[3,4]$. We report here the discovery and confirmation of 70 non-eclipsing BEER binaries in CoRoT data.

\section{Observations and data analysis}

We applied the BEER algorithm to the white lightcurves of the CoRoT fields LRc01, LRc02, LRc03, LRc04, and LRc05, similarly to the way it was done for Kepler lightcurves by [2], and selected a total of 481 candidates for RV follow-up from all five fields. 281 of the selected candidates were eventually observed by the AAOmega multi-object spectrograph on the Anglo-Australian Telescope (AAT).

The observations took place on seven consecutive nights starting on August 02, 2012, and for most of the observed candidates we got six to seven medium-resolution $(R \sim 10,000)$ AAOmega spectra. Observations and data reduction were performed similarly to previously reported works [6]. Table 1 lists the coordinates, magnitudes, photometric ephemeris, and amplitudes of the three BEER effects, for the observed candidates.

To derive RVs and errors from the observed spectra we calculated cross-correlation functions (CCFs) of the observed spectra with a set of synthetic Phoenix spectra [5]. To maximize the CCF peak values we selected for each star a template with an optimum set of spectral parameters (i.e. $T_{\text {eff }}$, $\log g,[\mathrm{~m} / \mathrm{H}]$, and $v \sin i)$. For double-lined spectra we used TODCOR [7], optimizing the primary and secondary templates and the flux ratio between them $(\alpha)$. The derived RVs, their uncertainties, and template parameters used to derive these RVs can be found at ftp://wise-ftp.tau.ac.il/pub/corotAAO.

a e-mail: levtalo@post.tau.ac.il

This is an Open Access article distributed under the terms of the Creative Commons Attribution License 4.0, which permits unrestricted use, distribution, and reproduction in any medium, provided the original work is properly cited. 
Table 1. Coordinates and photometric parameters of the BEER candidates observed at AAOmega*.

\begin{tabular}{lcccrrrrrr}
\hline \hline CoRoT ID & $\begin{array}{c}\text { RA } \\
(\mathrm{deg})\end{array}$ & $\begin{array}{c}\text { Dec } \\
(\mathrm{deg})\end{array}$ & $\begin{array}{c}\mathrm{V} \\
(\mathrm{mag})\end{array}$ & $\begin{array}{r}\text { Orbital } \\
\text { period } \\
\text { (day) }\end{array}$ & $\begin{array}{r}\text { Orbital } \\
\text { phase } \\
(\text { HJD-2451545) }\end{array}$ & $\begin{array}{r}\text { Ellipsoidal } \\
\text { amplitude } \\
(\mathrm{ppm})\end{array}$ & $\begin{array}{r}\text { Beaming } \\
\text { amplitude } \\
(\mathrm{ppm})\end{array}$ & $\begin{array}{r}\text { Reflection } \\
\text { amplitude } \\
(\mathrm{ppm})\end{array}$ & $\begin{array}{c}\text { Conf. } \\
\text { flag }\end{array}$ \\
\hline 105659320 & 280.4275 & 5.8974 & 14.8 & 0.70597 & 3171.931 & -2203 & 367 & 1497 & 1 \\
& & & & 0.00032 & 0.035 & 11 & 66 & 111 & \\
105962436 & 280.9442 & 5.8186 & \multirow{2}{*}{14.2} & 1.80188 & 3171.579 & -3563 & 220 & -677 & 1 \\
& & & & 0.00062 & 0.025 & 31 & 26 & 43 & \\
\hline
\end{tabular}

${ }^{*}$ Each line of parameters is followed by a line of uncertainties. Two of the table entries are shown here for guidance regarding its form and content. The table is available in its entirety at $\mathrm{ftp} / / /$ wise-ftp.tau.ac.il/pub/corotAAO.
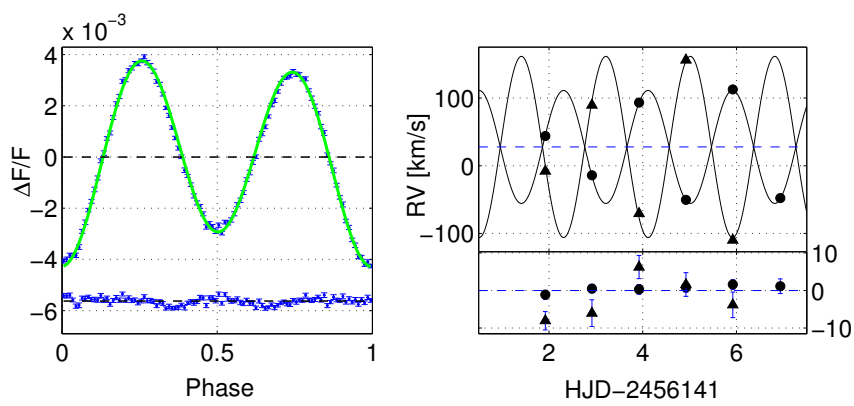

Fig. 1. Illustration of the discovery and confirmation process of the BEER SB2 105962436. Left: phase-folded lightcurve with the best-fit BEER model. Right: AAOmega RVs with the best-fit Keplerian model.

To separate between true BEER binaries and false alarms (FAs) we fitted the derived RVs with a circular Keplerian model, taking the BEER period and phase as priors, and applied $\chi^{2}$ - and $F$-statistics for the orbital solution. For double-line spectra we fitted a circular Keplerian model for the primary and secondary RVs separately, as well as fitting a circular Keplerian model of a double-line binary (SB2) for the two sets of RVs together, in both cases taking the BEER period and phase as priors. Figure 1 shows the phase-folded lightcurve with the best-fit BEER model, and the AAOmega RVs with the best-fit Keplerian model for one of the confirmed SB2s - CoRoT id 105962436.

In total, we confirmed the binarity of 70 non-eclipsing BEER candidates, with 18 of them being SB2s. The confirmed binaries are indicated in the rightmost column of Table 1.

\section{Conclusions}

Using RV follow-up observations with AAOmega, we have demonstrated for the first time the capability of the BEER algorithm to detect non-eclipsing short-period binaries in CoRoT lightcurves. We discovered 70 such binaries, with 18 of them being SB2s. These detections can help revealing the detection rate and the false-alarm statistics of the BEER algorithm applied to CoRoT data.

\section{References}

1. Faigler, S., Mazeh, T., MNRAS 415, (2011) 3921-3928

2. Faigler, S., Mazeh, T., Quinn, S. N., Latham, D. W., Tal-Or, L., ApJ 746, (2012) 185

3. Faigler, S., Tal-Or, L., Mazeh, T., Latham, D. W., Buchhave, L. A., ApJ 771, (2013) 26

4. Faigler, S., Mazeh, T., ApJ 800, (2015) 73

5. Hauschildt, P. H., Allard, F., Baron, E., ApJ 512, (1999) 377

6. Sebastian, D., Guenther, E. W., Schaffenroth, V., Gandolfi, D., et al., A\&A 541, (2012) 34

7. Zucker, S., Mazeh, T., ApJ 420, (1994) 806 